\title{
Tet-mediated covalent labelling of 5-methylcytosine for its genome-wide detection and sequencing
}

Liang Zhang ${ }^{1, \star}$, Keith E. Szulwach ${ }^{2, \star}$, Gary C. Hon ${ }^{3, \star}$, Chun-Xiao Song $^{1}$, Beomseok Park ${ }^{4}$, Miao Yu ${ }^{1}$, Xingyu Luํ, Qing Dai ${ }^{1}$, Xiao Wang ${ }^{1}$, Craig R. Street ${ }^{2}$, Huiping Tan ${ }^{2}$, Jung-Hyun Min ${ }^{4}$, Bing Ren ${ }^{3}$, Peng $\mathrm{Jin}^{2} \&$ Chuan $\mathrm{He}^{1}$

5-methylcytosine is an epigenetic mark that affects a broad range of biological functions in mammals. The chemically inert methyl group prevents direct labelling for subsequent affinity purification and detection. Therefore, most current approaches for the analysis of 5methylcytosine still have limitations of being either density-biased, lacking in robustness and consistency, or incapable of analysing 5-methylcytosine specifically. Here we present an approach, TAmC-Seq, which selectively tags 5-methylcytosine with an azide functionality that can be further labelled with a biotin for affinity purification, detection and genome-wide mapping. Using this covalent labelling approach, we demonstrate high sensitivity and specificity for known methylated loci, as well as increased $\mathrm{CpG}$ dinucleotide coverage at lower sequencing depth as compared with antibody-based enrichment, providing an improved efficiency in the 5-methylcytosine enrichment and genome-wide profiling.

\footnotetext{
${ }^{1}$ Department of Chemistry and Institute for Biophysical Dynamics, University of Chicago, 929 E 57th Street, Chicago, Illinois 60637, USA. ${ }^{2}$ Department of Human Genetics, Emory University School of Medicine, 615 Michael Street, Atlanta, Georgia 30322, USA. ${ }^{3}$ Ludwig Institute for Cancer Research, Department of Cellular and Molecular Medicine, UCSD, Moores Cancer Center, and Institute of Genome Medicine, University of California, San Diego School of Medicine, 9500 Gilman Drive, La Jolla, California 92093-0653, USA. ${ }^{4}$ Department of Chemistry, University of Illinois at Chicago, 845 West Taylor Street, Chicago, Illinois 60606, USA. * These authors contributed equally to this work. Correspondence and requests for materials should be addressed to C. H. (email: chuanhe@uchicago.edu)
} 
$\mathrm{D}$ NA methylation (5-methylcytosine, $5 \mathrm{mC}$ ) is a widely studied epigenetic mark. It is catalysed and maintained by a family of DNA methyltransferases in eukaryotes, and takes place at $\sim 3-6 \%$ of the total cytosines in human genomic DNA $^{1}$. DNA methylation could impact a broad range of biological processes in mammals, including gene expression, maintenance of genome integrity, parental imprinting, $\mathrm{X}$-chromosome inactivation and development ${ }^{2-4}$. Moreover, abnormal DNA methylation has been associated with various human diseases, in particular cancers ${ }^{5-7}$. Knowledge of DNA methylation patterns and distributions in the genome is clearly crucial for us to understand the biological role of $5 \mathrm{mC}$ in normal human biology and aetiology of diseases.

To date, three different strategies have been developed to profile and analyse genome-wide DNA methylation patterns (methylome) in eukaryotic cells ${ }^{8}$. Restriction enzyme-based approaches were the earliest strategy to be adapted for genomewide methylation analysis. With the use of restriction enzymes that are sensitive to DNA methylation at the recognition sequence, $5 \mathrm{mC}$ could be detected at selected sequences. However, these approaches are limited to the specific restriction sites in the genome. In the second strategy, DNA fragments containing $5 \mathrm{mC}$ are enriched using affinity-based capture, including the use of 5mC-binding proteins (MBD-Seq) and antibody-based approaches (for example, methylated DNA immunoprecipitation or MeDIP-Seq). Bisulphite sequencing is the third common strategy in which denatured DNA is treated with sodium bisulphite, such that non-modified cytosine is converted to uracil, whereas methylated cytosine is left intact, thus allowing for base-resolution detection of cytosine methylation. Bisulphite sequencing is the current "gold standard" for DNA methylation analyses, owing to its sensitivity, high resolution and quantitativeness. However, this strategy cannot distinguish $5 \mathrm{mC}$ from $5 \mathrm{hmC}^{9,10}$, which was recently discovered as yet another form of cytosine methylation along with formyl- $\mathrm{C}(5 \mathrm{fC})$ and carboxy-C $(5 \mathrm{caC})^{11,12}$. As a result, new approaches have been developed recently to distinguish $5 \mathrm{hmC}$ from $5 \mathrm{mC}$ with a single-base resolution ${ }^{13,14}$.

Although whole-genome bisulphite sequencing approaches have been developed ${ }^{15-17}$ and are increasingly adopted, affinitybased approaches, such as MBD-Seq and MeDIP-Seq, are still widely used, because of the low cost, in laboratories for genome-wide and loci-specific detection and/or profiling of $5 \mathrm{mC}$. However, existing approaches commonly exhibit methylcytosine density-dependent bias that leads to lack of information at hypomethylated CpGs and non-CpG-methylated regions ${ }^{18-20}$. Bisulphite sequencing, although providing the most accurate methylation information, can be costly and requires a relatively large amount of samples because of DNA degradation during bisulphite treatment. As a result, further technology development of a robust, efficient, unbiased approach for whole-genome methylation profiling is still highly desirable. The development of such an approach will aid the general community in consistent profiling of methylomes, and in developing disease-specific diagnoses as well as establishing biomarkers ${ }^{21,22}$.

Here we propose a new approach inspired by the discovery that $5 \mathrm{mC}$ can be oxidized by the iron(II)/ $\alpha$-ketoglutarate $(\alpha-\mathrm{KG})$ dependent dioxygenases, the Tet (Ten-eleven translocation) family proteins (Tet1, 2 and 3 ), to $5 \mathrm{hmC}$, which can be further converted to $5 \mathrm{fC}$ and $5 \mathrm{caC}$ in genomic DNA of mammalian cells ${ }^{11,12,23-25}$. We and others have invented chemical-labelling approaches to selectively tag $5 \mathrm{hmC}$ with functional groups, such as biotin, for robust affinity enrichment and sequencing without sequence and modification density bias ${ }^{26,27}$. We envisioned that chemical labelling of $5 \mathrm{hmC}$ could be combined with
Tet-mediated conversion of $5 \mathrm{mC}$ to $5 \mathrm{hmC}$ for a selective labelling of $5 \mathrm{mC}$ for genome-wide detection and profiling. In our new approach, we first protect $5 \mathrm{hmC}$ with a glucose, using T4 bacteriophage $\beta$-glucosyltransferase $(\beta$-GT)-mediated glucosylation of $5 \mathrm{hmC}$, which is a quantitative reaction ${ }^{26}$. We then employ the mouse Tet1 catalytic domain (residues 13672039 , named $\mathrm{mTet} 1$ here and after) to oxidize $5 \mathrm{mC}$ to $5 \mathrm{hmC}$, and simultaneously trap the newly generated $5 \mathrm{hmC}$ by $\beta$-GTmediated transfer of a modified glucose moiety (6- $\mathrm{N}_{3}$-glucose) to afford 6- $\mathrm{N}_{3}-\beta$-glucosyl-5-hydroxymethyl-cytosine $\left(\mathrm{N}_{3}\right.$ $5 \mathrm{gmC})^{26}$. Utilizing Huisgen cycloaddition (click) chemistry, a biotin tag (or any chemical tag) is then installed through the azide group of $\mathrm{N}_{3}-5 \mathrm{gmC}$ for selective, efficient and unbiased pulldown of the original $5 \mathrm{mC}$-containing DNA fragments for detection and genome-wide profiling (Fig. 1) ${ }^{26}$. We named this new approach as Tet-assisted $5 \mathrm{mC}$ sequencing (TAmC-Seq). We show, by applying TAmC-Seq to profile $5 \mathrm{mC}$ in several mouse and human cell lines, that TAmC-Seq provides a wider coverage of CpGcontaining genomic regions and captures a larger fraction of methylated CpGs with much fewer reads and less density bias compared with other affinity-enrichment methods.

\section{Results}

Coupling Tet oxidation with $\beta$-GT for $5 \mathrm{mC}$-specific labelling. We cloned, expressed and purified mTetl using a baculovirus expression system, according to the published procedure ${ }^{24}$. The enzyme activity was confirmed with in vitro activity assays (Methods and Supplementary Fig. S1) ${ }^{13}$. As indicated in the previous studies, the overall conversion of $5 \mathrm{mC}$ to $5 \mathrm{fC} / 5 \mathrm{caC}$ by mTet1 is not processive in that the step of $5 \mathrm{mC}$ oxidation to $5 \mathrm{hmC}$ is kinetically faster than the subsequent $5 \mathrm{hmC}$ oxidation ${ }^{12}$. This knowledge presents an opportunity to capture the newly generated $5 \mathrm{hmC}$ with an efficient $\beta$-GT-mediated labelling reaction (Fig. 1) ${ }^{26}$. We further confirmed that mTetl could recognize and oxidize $5 \mathrm{mC}$ in a model double-stranded DNA (dsDNA) that contains hemi- $5 \mathrm{mC}$, full- $5 \mathrm{mC}$ or hemi- $5 \mathrm{mC} /$ hemi$5 \mathrm{hmC}$ modification (Supplementary Fig. S2), ensuring that $5 \mathrm{mC}$ in various contexts in genomic DNA could be efficiently recognized and oxidized by mTet1 for further labelling. In addition, unlike $\mathrm{TAB}-\mathrm{Seq}$, which requires highly reactive mTet1 to fully convert $5 \mathrm{mC}$ to $5 \mathrm{caC}$ (ref. 13), most mTet 1 proteins expressed from insect cells are fully active to convert $5 \mathrm{mC}$ to $5 \mathrm{hmC}$ in high efficiency.

We tested our approach on a model dsDNA with a $5 \mathrm{mC}$ containing 12 mer oligonucleotide annealed to a complementary $16 \mathrm{mer}$ oligonucleotide containing a $5 \mathrm{hmC}$ modification (Fig. 2a). In the first step, we transferred a regular glucose from uridinediphosphoglucose to the $5 \mathrm{hmC}$ base using $\beta$-GT. The resulting $5 \mathrm{gmC}$ can no longer be oxidized or labelled as indicated, by treating the dsDNA with mTet1 under oxidation conditions (Fig. 2a). However, mTet1 oxidation efficiently converts the $5 \mathrm{mC}$ on the opposite strand to $5 \mathrm{hmC}$. To avoid the potential overoxidation of $5 \mathrm{mC}$ to $5 \mathrm{fC}$ and $5 \mathrm{caC}$ by mTet1, we developed a one-pot procedure (referred to as the one-pot mTet $1 / \beta-G T$ reaction), envisioning that the $5 \mathrm{hmC}$ generated from oxidation of $5 \mathrm{mC}$ could be immediately captured and labelled with $6-\mathrm{N}_{3}$-glucose by $\beta$-GT-mediated glucosylation, which could effectively prevent overoxidation of the newly generated $5 \mathrm{hmC}$. The new $\mathrm{N}_{3}-5 \mathrm{gmC}$ is then labelled with biotin via click chemistry. As shown in Fig. 2b, $\sim 90 \%$ hemi- $5 \mathrm{mC}$ on the model dsDNA could be successfully labelled with biotin in the one-pot mTet $1 / \beta$-GT reaction, whereas the sequential treatment of the same dsDNA with mTet 1 and then $\beta$-GT only afforded $\sim 20 \%$ of the labelling (Supplementary Fig. S3). 
a<smiles>Cc1cn([18OH])c(=O)nc1N</smiles>

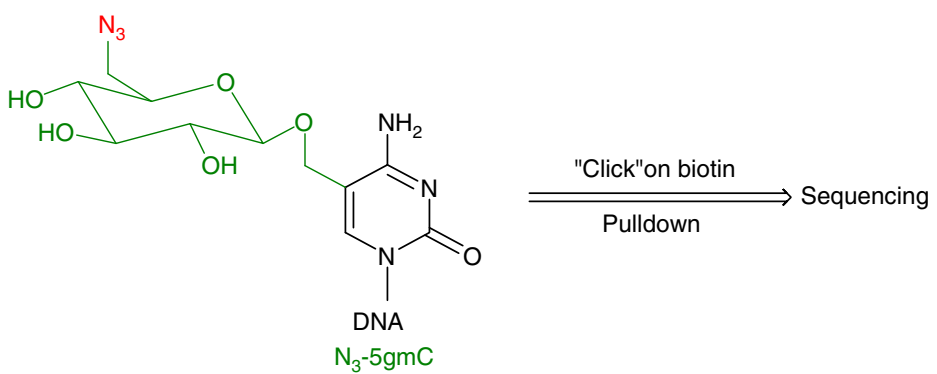

b

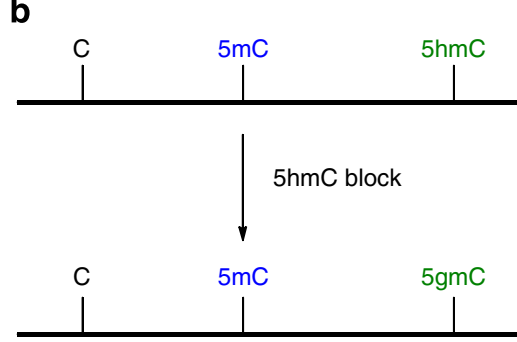<smiles>Cc1cn(C(C)(C)C)c(=O)nc1N</smiles><smiles>Nc1nc(=O)n([N+](=O)[O-])cc1CO</smiles><smiles></smiles><smiles>CS(=O)(=O)n1cc(COC2OCC(O)C(O)C(O)C2O)c(N)nc1=O</smiles>

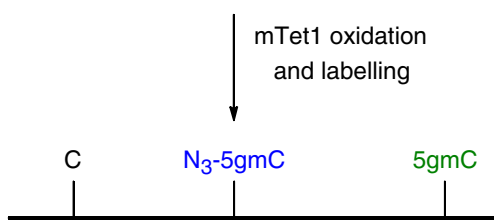<smiles>N#CC1COC(OCc2cn(S(N)(=O)=O)c(=O)nc2N)C(O)C(O)C1O</smiles>

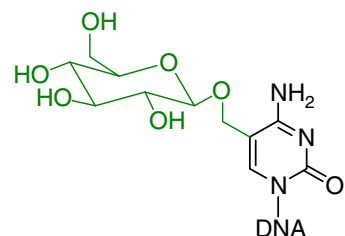

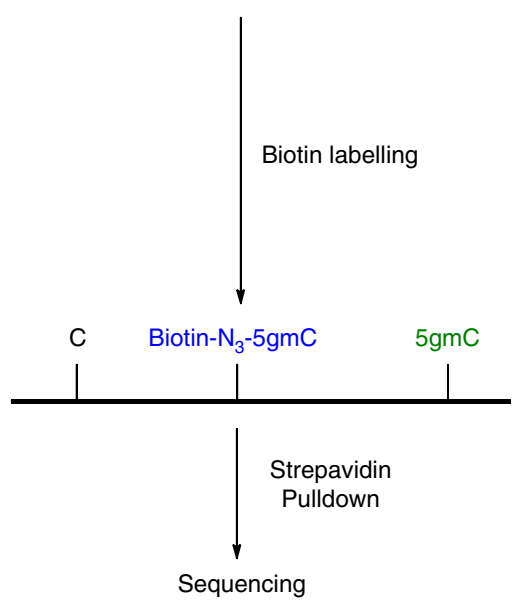

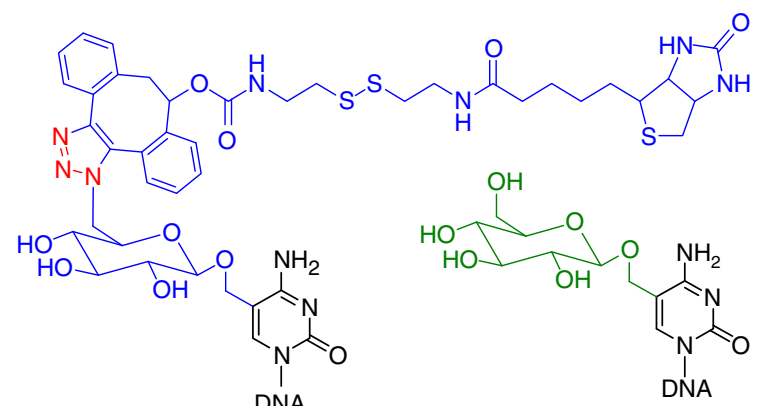

Figure 1 Schematic diagram of the selective labelling of $\mathbf{5 m C}$ in DNA. (a) $5 \mathrm{mC}$ can be converted to $5 \mathrm{hmC}$ via mTet1-catalysed oxidation, and then labelled with a modified glucose moiety (6- $\mathrm{N}_{3}$-Glucose) by $\beta$-GT-mediated glucosylation to afford $6-\mathrm{N}_{3}-\beta$-glucosyl-5-hydroxymethyl-cytosine ( $\mathrm{N}_{3}-5 \mathrm{gmC}$ ), which could be further labelled using click chemistry. (b) The endogenous $5 \mathrm{hmC}$ in genomic DNA can be protected by glycosylation with regular glucose. Using a one-pot mTet1/ $\beta$-GT protocol, $5 \mathrm{mC}$ is converted into $\mathrm{N}_{3}-5 \mathrm{gmC}$ in the presence of both mTet 1 and $\beta$-GT. The biotin moiety can then be installed for subsequent detection, affinity purification and sequencing.

Efficient and specific $5 \mathrm{mC}$ labelling in genomic DNA. We first applied the established approach to label and profile genomic DNA from J1 mouse embryonic stem cells (referred to as mESC here and after). The candidate genomic DNA was sonicated into small fragments ( $\sim 300-500 \mathrm{bp})$, and after protection of $5 \mathrm{hmC}$ with glucose, an appropriate amount of mTet1 was added to mediate $5 \mathrm{mC}$ oxidation. Using the one-pot $\mathrm{mTet} 1 / \beta$-GT protocol, $80-120$ pmol mTet1 converted and labelled most $5 \mathrm{mC}$ (cannot be detected by $5 \mathrm{mC}$-specific antibody) to biotin from $1 \mu \mathrm{g}$ genomic DNA with no overoxidized products (5fC and $5 \mathrm{caC}$, detected by antibodies specific against these two bases) detected by immunoblot (Fig. 2c, d). In contrast, the sequential 
a

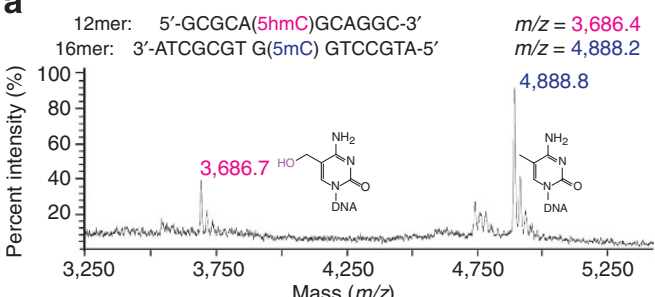

$\beta$-GT+ UDP-Glc $\downarrow \begin{aligned} & \Delta m / z_{\text {cal }}=162.0 \\ & \Delta m / z_{\text {obs }}=162.0\end{aligned}$

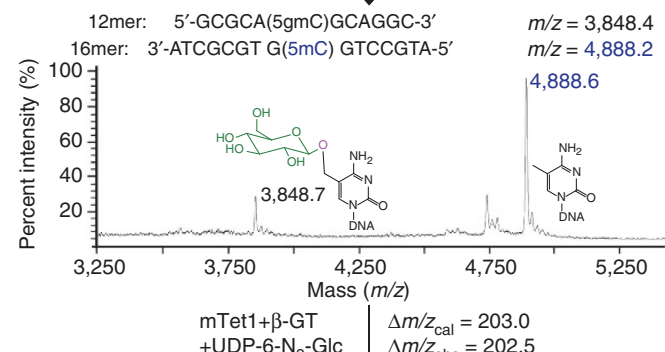

$\Delta m / z_{\text {cal }}=203.0$
$\Delta m / z_{\text {obs }}=202.5$

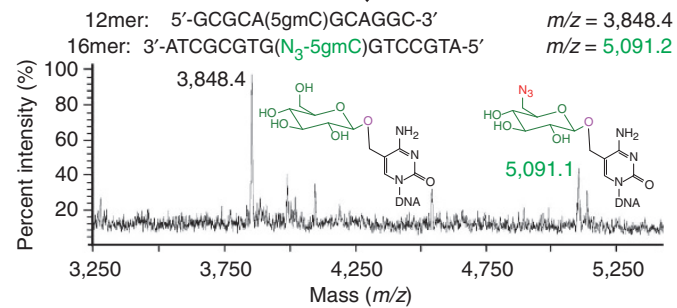

b

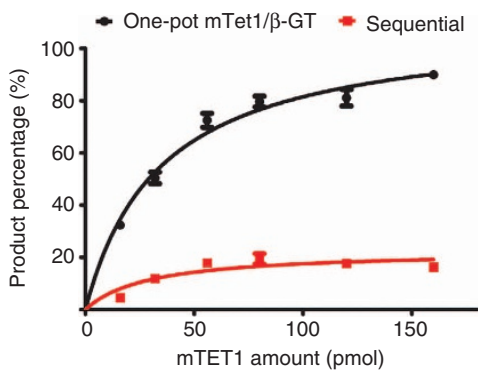

C
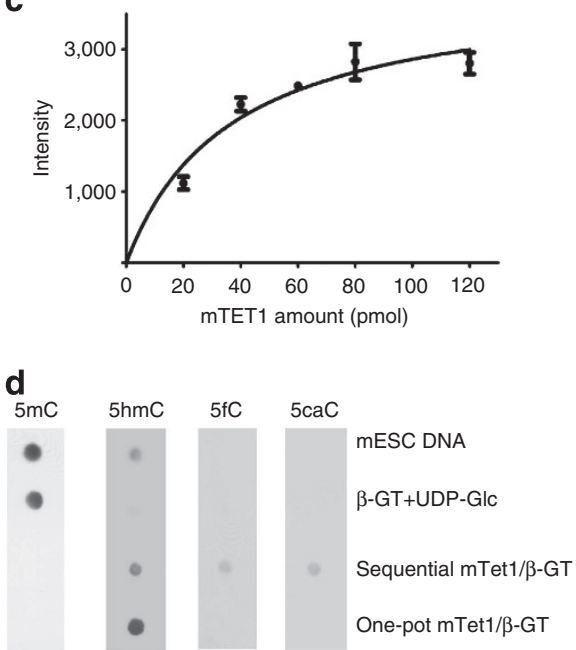

Figure 2 | Validation of the $\mathbf{5 m C}$-labelling approach using model dsDNA and genomic DNA. (a) Mass spectrometry characterization of the model reaction with a $5 \mathrm{mC}$ containing a $5 \mathrm{hmC}$-containing $12 \mathrm{mer} \mathrm{DNA}$, annealed to a $5 \mathrm{mC}$-containing $16 \mathrm{mer}$ DNA. The reactions were monitored by matrixassisted laser desorption/ionization-time of flight with the calculated molecular weight and observed molecular weight indicated. (b) Comparison of the $5 \mathrm{mC}$ conversion and labelling by using the one-pot mTet1/ $\beta$-GT method (black curve) and sequential mTet1/ $\beta$-GT method (red curve). $32 \mathrm{mer}$ dsDNA $(50 \mathrm{pmol})$ containing a single $5 \mathrm{mC}$ was used as the substrate, and various concentrations of recombinant mTet1 were used as indicated. The conversion ratio was evaluated by dot blot assay of the attached biotin. As shown in the figure, the one-pot mTet $1 / \beta-G T$ method labelled over $90 \%$ of $5 \mathrm{mC}$ in the model DNA. Error bars represent data with s.d. from triplicate experiments. (c) $5 \mathrm{mC}$ labelling on mESC genomic sample by using the one-pot mTet1/ $\beta$-GT method. Various concentrations of recombinant mTet1 were used as indicated. mTet1 protein ( $80-120$ pmol) labelled most $5 \mathrm{mC}$ in $1 \mu \mathrm{g}$ genomic DNA. Error bars represent data from triplicate experiments. (d) The labelled genomic DNA (mESC) products were validated by using antibodies against $5 \mathrm{mC}(1 \mu \mathrm{g}), 5 \mathrm{fC}(150 \mathrm{ng})$ and $5 \mathrm{caC}(150 \mathrm{ng})$, and dot blot assay for $5 \mathrm{hmC}(150 \mathrm{ng})$. The one-pot mTet $1 / \beta$-GT method converted most $5 \mathrm{mC}$ to $5 \mathrm{hmC}$ for subsequent labelling, with no overoxidation products detected.

mTet $1 / \beta$-GT treatment led to noticeable amounts of $5 \mathrm{fC}$ and $5 \mathrm{caC}$ generated (Fig. $2 \mathrm{~d}$ ). The conversion ratio of $5 \mathrm{mC}$ in $\mathrm{mESC}$ DNA is further confirmed by using liquid chromatography-mass spectrometry (LC-MS), which revealed that the endogenous $5 \mathrm{hmC}$ in genomic DNA were fully protected, and over $90 \% 5 \mathrm{mC}$ were converted and labelled to $\mathrm{N}_{3}-5$ gmC (Supplementary Fig. S4 and Supplementary Tables S1 and S2). Furthermore, we have also confirmed that the glucose labelling of $5 \mathrm{hmC}$ is not reversible under our assay conditions (Supplementary Fig. S5) ${ }^{28}$. The biotin-labelled genomic DNA samples were subsequently processed by following the previously developed streptavidin pulldown protocol $^{29}$.

Application of TAmC-Seq for genome-wide $5 \mathrm{mC}$ profiling. Previous comparison of approaches for mapping DNA methylation in ES cells highlighted the advantage of MeDIP-Seq for capturing a relatively large number of $\mathrm{CpG}$ genome-wide, with relatively few reads, making this a cost-effective approach despite known biases associated with it ${ }^{20}$. Having established TAmC-Seq as both sensitive and specific for $5 \mathrm{mC}$ on model DNA, as well as its effectiveness on genomic DNA, we sought to further assess the utility of this new approach in determining the genome-wide distribution of $5 \mathrm{mC}$. We therefore applied it to mESC genomic DNA. This experiment allowed further direct comparison of TAmC-Seq with previously published MeDIP-Seq ${ }^{30}$, and quantitative assessment of $5 \mathrm{mC}$ enriched by these two approaches.

Comparison of read densities indicated that TAmC-Seq provides a highly similar pattern of enrichment as compared with MeDIP-Seq. Indeed, normalized count data in genome-wide bins $(10 \mathrm{~kb})$ displayed a correlation coefficient of $0.81\left(R^{2}\right)$ between TAmC-Seq and MeDIP-Seq (Fig. 3a). Likewise, readnormalized binary calling of methylated regions with increasing read-coverage thresholds (see Methods) showed that TAmC-Seq could account for $>94 \%$ of the genomic sequences identified as methylated by MeDIP-Seq, with a minimum read depth of five, confirming the specificity of TAmC-Seq for capturing $5 \mathrm{mC}$ (Fig. 3b). The same regions were further validated by comparing with conventional whole-genome bisulphite sequencing data ${ }^{13}$, which revealed an average of $78.34 \%$ methylation at $\mathrm{CpG}$ dinucleotides (Supplementary Table S3). We also found that TAmC-Seq generally exhibits a more even coverage than MeDIPSeq (Supplementary Figs S6 and S7). This observation suggests that, although specifically interrogating $5 \mathrm{mC}$, TAmC-Seq is able to capture a larger fraction of methylated $\mathrm{CpGs}$ with reduced 
a

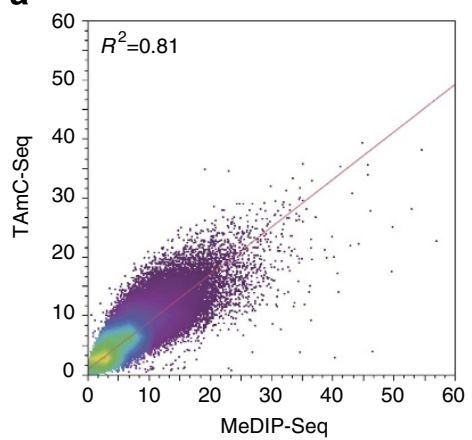

C

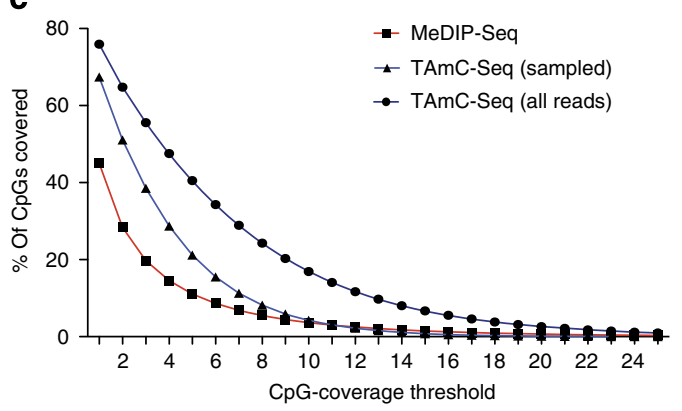

e

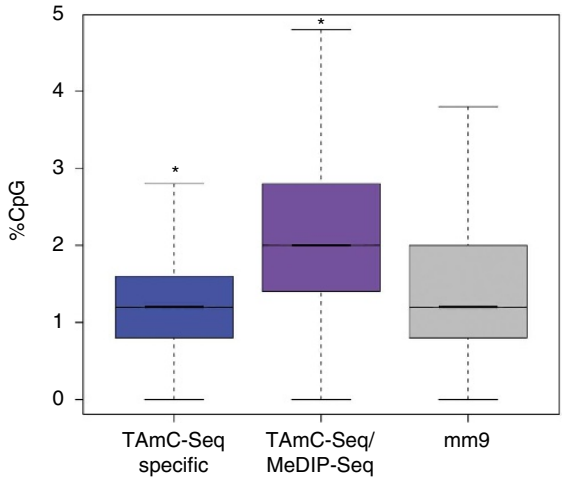

b

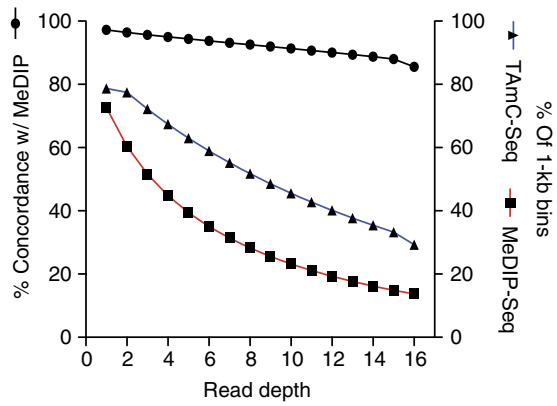

d

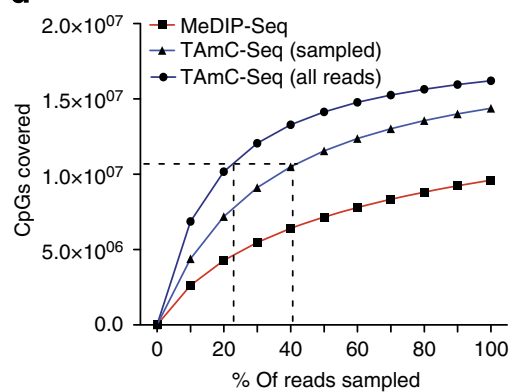

$\mathbf{f}$

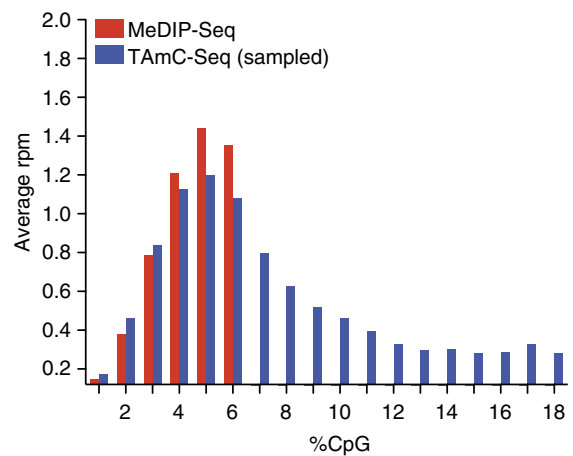

Figure 3 | Genome-wide comparison of MeDIP-Seq and TAmC-Seq. (a) Genome-wide correlation between TAmC-Seq and MeDIP-Seq (10 kb bins, reads per million). (b) Concordance and genomic coverage of TAmC-Seq and MeDIP-Seq with varying read depth thresholds. Percentage concordance (left $y$ axis, black line), shows the percentage of $1 \mathrm{~kb}$ bins with the given read depth threshold in MeDIP-Seq that agree with TAmC-Seq. Right $y$ axis shows the percentage of $1 \mathrm{~kb}$ bins covered at varying read depth thresholds per approach (TAmC-Seq, blue line; MeDIP-Seq, red line). Comparisons were performed using equal numbers of reads. (c) The fraction of $\mathrm{CpGs}$ covered genome-wide as a function of the depth of coverage for TAmC-Seq and MeDIP-Seq. TAmC-Seq reads were randomly sampled to match the number of reads in MeDIP-Seq. (d) The number of CpGs covered genome-wide by TAmC-Seq and MeDIP-Seq with varying fractions of reads used. The horizontal dotted line indicates $50 \%$ of all CpGs genome-wide. Vertical lines indicate the percentage of reads required to cover $50 \%$ of all $\mathrm{CpGs}\left(K_{\mathrm{CpG}-\mathrm{Seq}}\right.$ ) with all TAmC-Seq reads or TAmC-Seq reads randomly sampled to match the number of MeDIP-Seq reads. (e) Mean \%CpG content in TAmC-Seq-specific $1 \mathrm{~kb}$ bins, MeDIP-Seq/TAmC-Seq-concordant 1kb bins and NCBI37v1/mm9;

${ }^{\star} P<0.0001$, Z-test. (f) The distribution of $5 \mathrm{mC}$ signals (average reads per million, rpm) derived from MeDIP-Seq or TAmC-Seq for $1 \mathrm{~kb}$ bins stratified by $\% \mathrm{CpG}$ content.

density-related bias, resulting in an effective spreading of reads more evenly throughout the methylated regions.

To quantify the comparative efficiency of capture by each approach, we measured the number of $\mathrm{CpG}$ dinucleotides covered genome-wide as a function of the number of reads sequenced. With an equivalent number of reads, TAmC-Seq is capable of covering 22.4\% more CpG dinucleotides than MeDIPSeq (Fig. 3c). When using the full set of TAmC-Seq reads, $76 \%$ of all CpGs were covered, approaching the estimated percentage of methylated cytosines observed in mouse ES cells $(\sim 80 \%)$ as determined by conventional bisulphite sequencing (Fig. $3 \mathrm{c})^{31}$. As an overall measure of efficiency, we next determined the number of reads required to cover $50 \%$ of all $\mathrm{CpG}$ dinucleotides
$\left(K_{\mathrm{CpG}-\mathrm{Seq}}\right)$, assuming that greater than this fraction are methylated in mouse ES cells ${ }^{31}$. $K_{\mathrm{CpG}-\mathrm{Seq}}$ for TAmC-Seq was $7.7 \times 10^{6}$ reads $(\sim 41 \%$ of total), whereas MeDIP-Seq failed to achieve $50 \%$ coverage with an equivalent number of reads (Fig. 3d). When utilizing the full set of TAmC-Seq reads $\left(3.5 \times 10^{7}\right), \quad K_{\mathrm{CpG}-\mathrm{Seq}}$ reached $22.7 \%$ (Fig. $\left.3 \mathrm{~d}\right)$. These results indicate that TAmC-Seq is able to enrich methylated CpGs more effectively than MeDIP-Seq, and demonstrate the increased efficiency of the approach for specifically interrogating $5 \mathrm{mC}$.

The primary drawback of the current affinity-based $5 \mathrm{mC}$ enrichment procedures is that they often exhibit $\mathrm{CpG}$ density bias, which can vary depending on antibody/protein source and the concentration of salts used during immunoprecipitation 
and/or elution. As a chemical-based approach that takes advantage of covalent connection and the high-affinity biotin/streptavidin enrichment, TAmC-Seq offers the potential to reduce the impact of such variables. To compare the relative biases associated with TAmC-Seq and MeDIP-Seq, we first determined \%CpG content within 1-kb bins concordant among each approach, as well as those captured specifically by TAmC-Seq, but not MeDIP-Seq, at readcoverage threshold of $\geq 5$, representing $94 \%$ of MeDIP-Seq 1-kb bins (Fig. 3e). Indicative of enrichment for $5 \mathrm{mC}$ by both approaches, concordant regions have significantly higher $\% \mathrm{CpG}$ content relative to the genome-wide mean $(P<0.0001, Z$-test). However, at regions specific to TAmC-Seq, the $\mathrm{CpG}$ percentage content is significantly lower than the genomic mean $(P<0.0001$, $Z$-test). This observation suggests that although TAmC-Seq captured a significant fraction of methylated regions detected by MeDIP-Seq, it also recovered additional loci with a lower percentage of $\mathrm{CpG}$ content. TAmC-Seq-specific regions were confirmed as methylated by comparing with the whole-genome bisulphite sequencing data ${ }^{13,31}$, showing $76.34 \%$ methylation of CpG dinucleotides (Supplementary Table S3). To further examine the average $5 \mathrm{mC}$ signals generated by TAmC-Seq and MeDIP-Seq at regions with varying percentage of $\mathrm{CpG}$ content, we also stratified genome-wide $1 \mathrm{~kb}$ bin read counts (reads per million) by $\% \mathrm{CpG}$ content. We found that TAmC-Seq is able to capture a wider range of $\mathrm{CpG}$ content than that of MeDIP-Seq, for which the distribution reflected the $\mathrm{CpG}$ content previously observed for MeDIP-Seq and MBD-Seq in mammalian genomes ${ }^{20,32}$ (Fig. 3f). Together, these data demonstrate a high concordance between TAmC-Seq and MeDIP-Seq, with TAmC-Seq providing additional access into the methylome compared with MeDIP-Seq.

Global comparison of methods that map DNA methylation. Next, we further compared the performance of TAmC-Seq with MethylC-Seq and MeDIP-Seq, two common approaches for DNA methylation mapping. We performed TAmC-Seq in human mammary epithelial cells (HMEC) and the breast cancer cell line HCC1954, and compared these maps with those previously generated by bisulphite sequencing and MeDIP-Seq ${ }^{33,34}$. After mapping and PCR duplicate filtering, the number of usable reads from TAmC-Seq and MeDIP-Seq were comparable (TAmC $_{\text {HMEC }}$ $=6.5$ million, $\mathrm{MeDIP}_{\mathrm{HMEC}}=6.3$ million, $\mathrm{TAmC}_{\mathrm{HCC} 1954}=7.1$ million).

Previously, using bisulphite sequencing, we had observed large domains of hypomethylation in HCC1954 compared with HMEC. This is clearly recapitulated by TAmC-Seq (Fig. 4a). At greater resolution, TAmC-Seq and bisulphite sequencing are also concordant; both approaches clearly reveal that the promoter of the DACH1 tumour suppressor is methylated, that the $\mathrm{CpG}$ island near the KLF5 promoter is unmethylated, and that KLF12 has an unmethylated $\mathrm{CpG}$ island but with methylated shores.

We next compared the performance of the affinity-based approaches with bisulphite sequencing. One way to assess the relative performance is to compute the correlation of two assay results throughout the genome. We partitioned the genome into non-overlapping 10-kb bins, measured DNA methylation from both affinity approaches as RPKM (reads per million base pairs mapped per $\mathrm{kb}$ of model) and plotted DNA methylation levels relative to MethylC-Seq (Supplementary Fig. S8). TAmC-Seq in HCC1954 reveals that the approach can recover global hypomethylation. Although the results for TAmC-Seq and MeDIP-Seq in HMEC cells are comparable, we observed a greater dynamic range in TAmC-Seq, which we discuss below.

Although DNA methylation is found throughout the genome, its most recognized role is as a mark of gene repression when found at promoters. Thus, a key performance metric of any approach to map DNA methylation is the ability to distinguish methylated from unmethylated promoters. To assess this performance, we compared the two affinity-based approaches with MethylC-Seq at promoters, where promoters are partitioned by $\mathrm{CpG}$ content (L/I/H = low/intermediate/high) (Fig. 4b, c). For all three examples, we observed a positive correlation between affinity-based and bisulphite-based DNA methylation. For all classes of promoters in HMEC cells, TAmC-Seq correlated better with bisulphite sequencing than MeDIP-Seq (low CpG: $R_{\mathrm{TAmC}}=0.18>0.15=R_{\mathrm{MeDIP}}$; intermediate $\mathrm{CpG}: R_{\mathrm{TAmC}}=0.67$ $>0.49=R_{\mathrm{MeDIP}} ; \quad$ high $\quad$ CpG: $\quad R_{\mathrm{TAmC}}=0.40>0.37=R_{\mathrm{MeDIP}}$. Furthermore, in agreement with the global analysis above, it appears that the dynamic range of TAmC-Seq is greater than that of MeDIP-Seq. To quantify this observation, we examined the affinity enrichment for lowly methylated (\%CpG $\leq 25 \%)$ and highly methylated (\%CpG $>75 \%$ ) promoters (Fig. 4c), as determined by bisulphite sequencing. We observe that the median level of TAmC-Seq enrichment is between 32.7 and $91.0 \%$ greater than that of MeDIP-Seq $\left(P_{\text {high }} \mathrm{CpG}=0.0035\right.$; $P_{\text {intermediate }} \mathrm{CpG}=8.0 \mathrm{E}-47 ; P_{\text {low }} \mathrm{CpG}=1.1 \mathrm{E}-39$, Wilcoxon rank sum test). These results suggest the TAmC-Seq dynamic range is greater than that of the MeDIP-Seq.

To systematically compare the ability of TAmC-Seq and MeDIP-Seq to distinguish methylated from unmethylated promoters, we first focused on a curated set of promoters that are lowly or highly methylated in HMEC according to bisulphite sequencing. We identified 1,388 promoters that are consistently highly methylated ( $>75 \%$ mCG) within and around the CpG island/transcription start site (TSS) and 7,621 promoters that are consistently lowly methylated ( $\leq 25 \%$ mCG; Fig. 5a). We then utilized a random forest to classify promoters as methylated or unmethylated, given only affinity enrichment. MeDIP-Seq gives a higher classification error rate than TAmC-Seq (Fig. 5b), indicating that TAmC-Seq enrichment is a better predictor of DNA methylation than the MeDIP-Seq enrichment. Extending this analysis to all promoters, we made similar observations (Fig. 5c). For all grades of CpG content, MeDIP-Seq consistently performs worse than TAmC-Seq for the task of classifying lowly and highly methylated promoters.

Finally, having explored the performance of TAmC-Seq in each cell line independently, we next examined its performance across multiple samples by comparing HMEC with HCC1954. At promoters, changes of DNA methylation observed from TAmCSeq are positively correlated with changes in absolute DNA methylation as measured by bisulphite sequencing (Fig. 5d). Employing a random forest to classify hypermethylated $\left(\% \mathrm{mCG}_{\mathrm{HCC} 1954}-\% \mathrm{mCG}_{\mathrm{HMEC}} \geq 50 \%\right)$ and hypomethylated (\% $\left.\mathrm{mCG}_{\mathrm{HCC} 1954}-\% \mathrm{mCG}_{\mathrm{HMEC}} \leq-50 \%\right)$ promoters, we found that TAmC-Seq approaches $75 \%$ accuracy for low- and intermediate-CpG promoters, and up to $85 \%$ accuracy for high CpG-content promoters (Fig. 5e). This ability to gauge cell-type specific DNA methylation at promoters of varying CpG content indicates that TAmC-Seq performance is relatively sequence independent.

\section{Discussion}

In summary, affinity-based enrichment of methylated DNA, when coupled with high-throughput sequencing, offers an effective approach for assessing DNA methylation on the genomic scale. The development of $5 \mathrm{mC}$-specific antibodies has made possible genomic DNA methylation profiling in various biological systems. However, the primary pitfall associated with $5 \mathrm{mC}$ immunoprecipitation is the methyl-CpGdensity-dependent bias, which ultimately results in missing certain portions of the methylome. In addition, various factors 

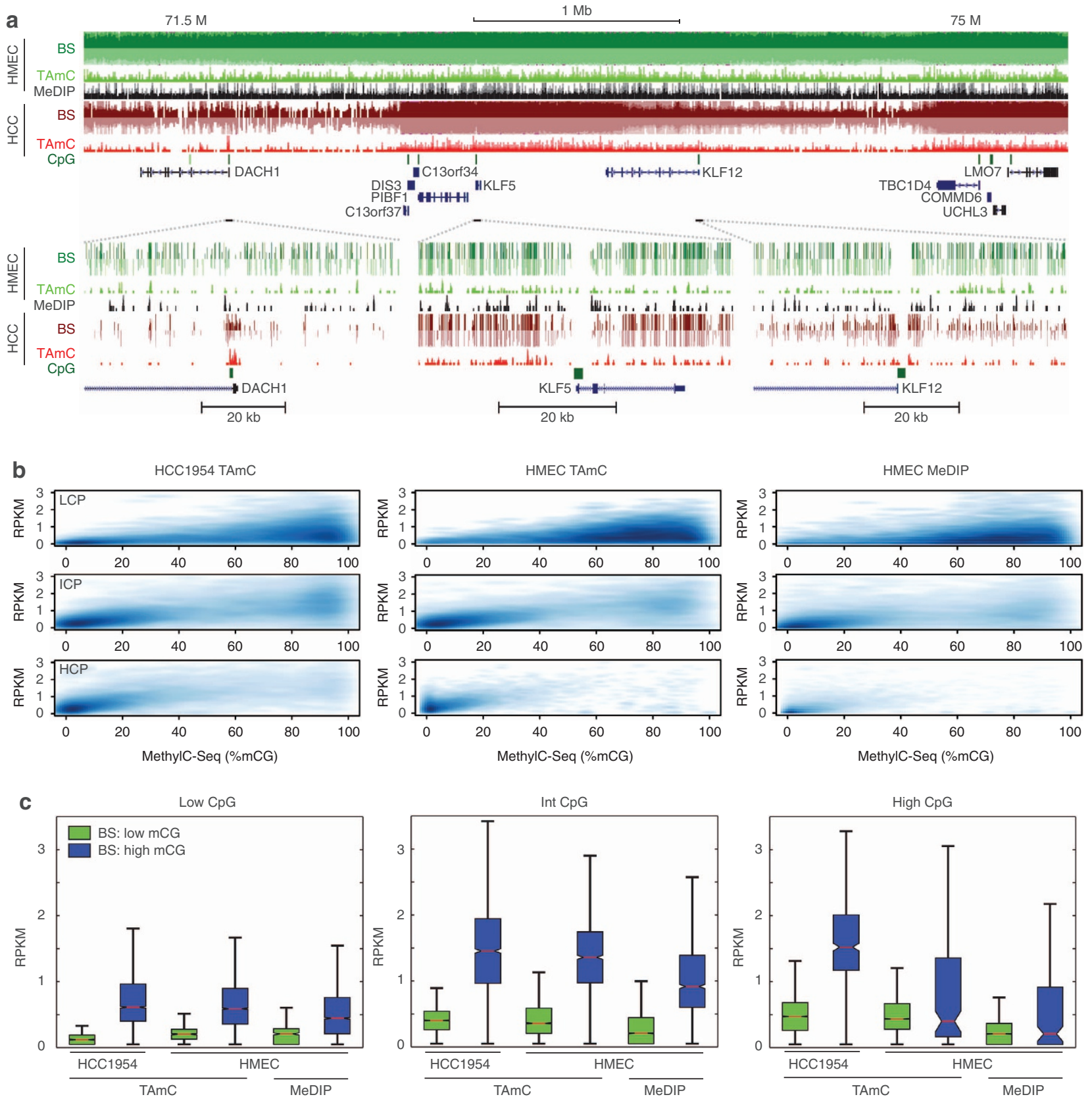

Figure 4 | Comparison of affinity approaches with bisulphite sequencing. (a) UCSC Genome Browser snapshots of TAmC-Seq, MeDIP-Seq and methylC-Seq near the DACH1 tumour suppressor locus. (b) Density plots of affinity enrichment compared with bisulphite sequencing at low CpG promoters (LCP, top), intermediate CpG promoters (ICP, middle) and high CpG promoters (HCP, bottom). (c) The distribution of affinity enrichment at promoters with low methylation ( $\leq 25 \% \mathrm{mCG}$, green) and high methylation ( $>75 \%$, blue), as determined by bisulphite sequencing.

could contribute to inconsistency of results obtained from antibody-based experiments.

Here we report an enzymatic strategy to specifically modify the inert methyl group of $5 \mathrm{mC}$, which utilizes a covalent linkage and a high-affinity biotin/streptavidin interaction to label and enrich $5 \mathrm{mC}$ in an unbiased manner. We found that TAmC-Seq is highly sensitive and specific to $5 \mathrm{mC}$, and captures a larger fraction of CpG dinucleotides with far fewer reads than MeDIP-Seq. Furthermore, TAmC-Seq provides a wider range of access to genomic regions with varying $\mathrm{CpG}$-dinucleotide frequencies, reducing CpG-density-dependent bias compared with that of MeDIP-Seq. In addition, the covalent strategy ensures the robustness of the approach for reliable profiling and/or locispecific pulldown and interrogation of DNA methylation without resorting to the more costly bisulphite-sequencing approach. TAmC-Seq thereby offers a consistent and robust tool to facilitate effective enrichment and epigenomic profiling of DNA methylation.

\section{Methods}

Recombinant mTET1 expression and purification. The catalytic domain of Mouse Tet1 gene (GU079948) was cloned into BssHII and NotI sites of the N-terminal Flag-tagged pFastBac Dual vector (Invitrogen) and expressed in Bac-toBac baculovirus insect cell expression system. The recombinant Flag-mTet1 (residues 1367-2039) was purified according to the published procedure (Supplementary Fig. S1a, b) ${ }^{13}$.

Recombinant $\beta$-GT expression and purification. The recombinant $\beta$-GT was expressed and purified following the previous protocol ${ }^{26}$. 
a

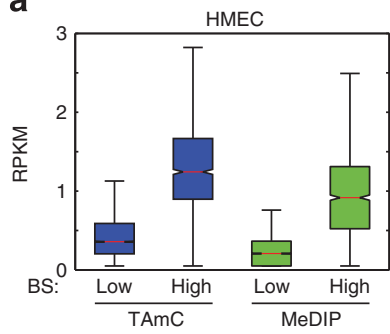

C

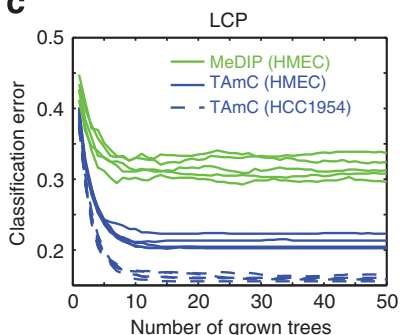

d

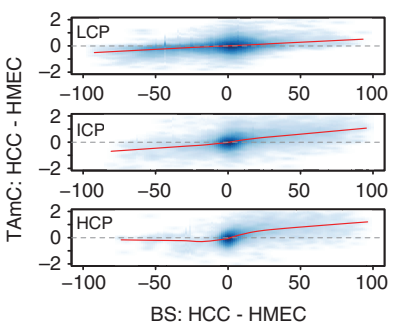

b
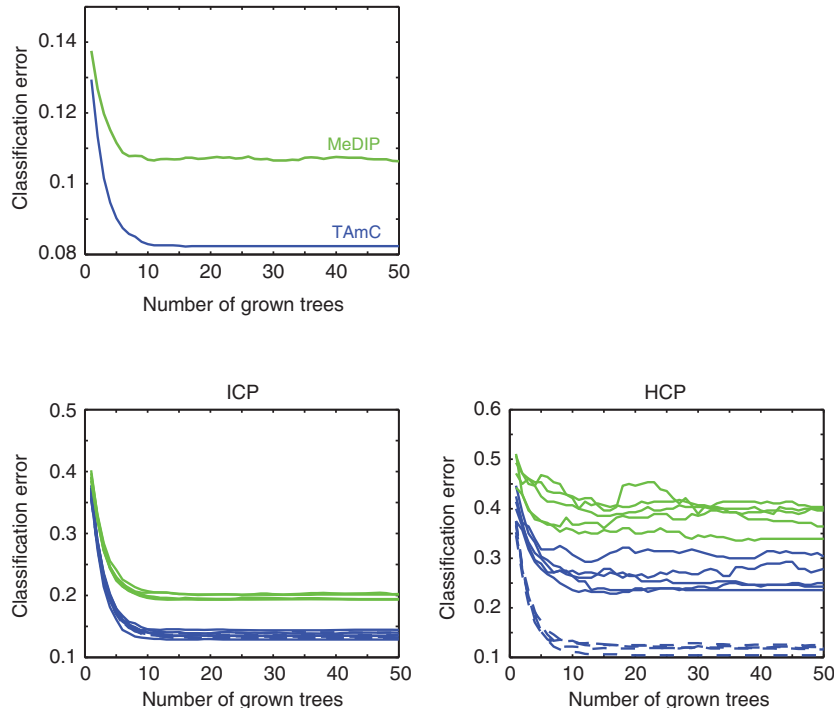

e

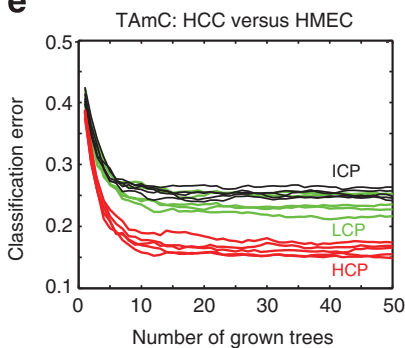

Figure 5 | Classification comparison of TAmC-Seq and MeDIP-Seq. (a) The distribution of TAmC-seq and MeDIP-seq enrichment for a set of curated promoters that are consistently highly methylated ( $>75 \% \mathrm{mCG}$ ) within and around the CpG island/TSS, or consistently lowly methylated ( $\leq 25 \% \mathrm{mCG}$ ). (b) Random forest classification error in distinguishing methylated from unmethylated curated promoters in a. (c) Random forest classification error in distinguishing methylated from unmethylated promoters, for different $\mathrm{CpG}$ densities. An equal number of methylated and unmethylated promoters were randomly sampled, and subsequently classified for each $\mathrm{CpG}$ density class. Each line represents a different random sampling of promoters. The high variance of HCPs is due to the small number of methylated high-CpG promoters in HMEC. (d) A density plot of bisulphite change (\%mCG(HCC1954)-\%mCG(HMEC)) compared with TAmC change (RPKM(HCC1954)-RPKM(HMEC)) for promoters of various CpG content. The red line is the lowest weighted curve; RPKM, reads per million base pairs mapped per kb of model. (e) Random forest classification error in distinguishing hypermethylated from hypomethylated promoters for different $\mathrm{CpG}$ densities. An equal number of hypermethylated and hypomethylated promoters were classified for each CpG density class. Each line represents a different random sampling of promoters.

Oligonucleotide synthesis. $12 \mathrm{mer}, 16 \mathrm{mer}, 32 \mathrm{mer}$ and $44 \mathrm{mer}$ oligonucleotides containing single $\mathrm{CpG}$ with modified cytosine $(5 \mathrm{mC}$ or $5 \mathrm{hmC})$ were prepared by incorporating the phosphoramidite $(5 \mathrm{mC}$ and $5 \mathrm{hmC})$ at the desired position during solid-phase synthesis. The reagents and phosphoramidites $(5 \mathrm{mC}$ and $5 \mathrm{hmC}$ ) were purchased from Glen Research. All synthetic oligonucleotides were further purified with denaturing polyacrylamide gel electrophoresis. The oligonucleotides containing normal bases were purchased from Operon.

Synthesis of UDP-6-N $\mathbf{N}_{\mathbf{3}}$-UDP. UDP-6- $\mathrm{N}_{3}$-UDP is synthesized by following the previous protocol ${ }^{26}$.

Recombinant $\mathbf{m T e t 1}$ in vitro activity assay. Various concentration of recombinant mTet 1 and 20 pmol 12/16mer dsDNA with internal 5-position methylated cytosine on $12 \mathrm{mer}$ DNA were added into the $20 \mu \mathrm{l}$ reaction mixture containing $50 \mathrm{mM}$ HEPES (pH 8) $75 \mu \mathrm{M} \mathrm{Fe}\left(\mathrm{NH}_{4}\right)_{2}\left(\mathrm{SO}_{4}\right)_{2}, 2 \mathrm{mM}$ ascorbic acid and $1 \mathrm{mM} \alpha$ $\mathrm{KG}$ for $1 \mathrm{~h}$ at $37^{\circ} \mathrm{C}$. The reaction products were then validated by matrix-assisted laser desorption/ionization-time of flight (Supplementary Fig. S1c). Anti-5mC antibody was obtained from Epigentek (Cat: A-1014). Antibodies against 5fC and $5 \mathrm{caC}$ were obtained from Active Motif (Cat: 61223 and 61225, respectively). Synthetic standards were used to quantify $5 \mathrm{mC}$ and $5 \mathrm{hmC}$ in activity assays ${ }^{26}$.

Recombinant $\mathbf{m}$ Tet1 substrate-selectivity assay. mTet $1(20 \mathrm{pmol}), \beta$-GT $(40 \mathrm{pmol})$ and $32 / 44 \mathrm{mer}(20 \mathrm{pmol}) \mathrm{dsDNA}$ with desired modified cytosine $(\mathrm{C}, 5 \mathrm{mC}$ or $5 \mathrm{hmC}$ ) were added into the $30 \mu \mathrm{l}$ reaction mixture containing $50 \mathrm{mM}$ HEPES (pH 8), $75 \mu \mathrm{M} \mathrm{Fe}\left(\mathrm{NH}_{4}\right)_{2}\left(\mathrm{SO}_{4}\right)_{2}, 2 \mathrm{mM}$ ascorbic acid, $1 \mathrm{mM} \alpha$-KG, $10 \mathrm{mM} \mathrm{MgCl}$,
$1 \mathrm{mM}$ dithiothreitol and $100 \mu \mathrm{M}$ UDP-6- $\mathrm{N}_{3}$-Glucose for $1 \mathrm{~h}$ at $37^{\circ} \mathrm{C}$. The DNA products were then purified using Qiagen DNA purification kit, and subsequently mixed with $150 \mu \mathrm{M}$ Dibenzylcyclooctyne-Fluor (Click Chemistry Tools Bioconjugate Technology Company) for $2 \mathrm{~h}$ at $37^{\circ} \mathrm{C}$. The labelled products were purified with the Qiagen purification kit, and $200 \mathrm{ng}$ was loaded to $16 \%$ urea-denatured acrylamide gels to separate the annealed strands. The gel was first scanned under $563 \mathrm{~nm}$ and the fluorescence was detected at $582 \mathrm{~nm}$, and then stained with Syber Green (Supplementary Fig. S2).

Recombinant mTet1- and $\beta$-GT-based chemical labelling of DNA. $\beta$-GT $(40 \mathrm{pmol})$ and $3 \mu \mathrm{g}$ sonicated mESC genomic DNA were added into the $30 \mu \mathrm{l}$ reaction mixture containing $50 \mathrm{mM}$ HEPES (pH 8), $25 \mathrm{mM} \mathrm{MgCl}_{2}$ and $300 \mu \mathrm{M}$ UDP-Glucose for $1 \mathrm{~h}$ at $37^{\circ} \mathrm{C}$. The product was purified using the Qiagen DNA purification kit. Subsequently, $160 \mathrm{pmol}$ mTet1, $80 \mathrm{pmol} \beta$-GT and $2 \mu \mathrm{g}$ treated genomic DNA were added into the $50 \mu \mathrm{l}$ reaction mixture containing $50 \mathrm{mM}$ HEPES ( $\mathrm{pH}$ 8), $75 \mu \mathrm{M} \mathrm{Fe}\left(\mathrm{NH}_{4}\right)_{2}\left(\mathrm{SO}_{4}\right)_{2}, 2 \mathrm{mM}$ ascorbic acid, $1 \mathrm{mM} \alpha-\mathrm{KG}, 10 \mathrm{mM}$ $\mathrm{MgCl}_{2}, 1 \mathrm{mM}$ dithiothreitol and $150 \mu \mathrm{M}$ UDP-6- $\mathrm{N}_{3}$-Glucose for $1 \mathrm{~h}$ at $37^{\circ} \mathrm{C}$. The DNA product was then purified and directly used in click reaction.

Huisgen cycloaddition reaction and pulldown. The Huisgen cycloaddition (click) reaction and pulldown was processed according to the previous protocol ${ }^{26}$ (Supplementary Methods).

Quantitative analysis of $\mathbf{5 m C}$ oxidation using L--MS/MS. mESC genomic DNA $(1 \mu \mathrm{g})$ with different treatment protocols (as indicated) was digested by using 
nuclease P1 (2U) in $30 \mu$ of buffer containing $25 \mathrm{mM}$ of $\mathrm{NaCl}$ and $2.5 \mathrm{mM}$ of $\mathrm{ZnCl}_{2}$ at $37^{\circ} \mathrm{C}$ for $2 \mathrm{~h}$, followed by the addition of $\mathrm{NH}_{4} \mathrm{HCO}_{3}(1 \mathrm{M}, 3 \mu \mathrm{l})$ and Venom phosphodiesterase $(0.001 \mathrm{U})$ and $2 \mathrm{~h}$ incubation at $37^{\circ} \mathrm{C}$. After an additional incubation at $37^{\circ} \mathrm{C}$ for $2 \mathrm{~h}$ with alkaline phosphatase $(0.5 \mathrm{U})$, the solution was diluted twofold, and $10 \mu \mathrm{l}$ of the solution was injected into LC-MS/MS. The nucleosides were separated by reverse-phase ultra-performance LC on a C18 column, with online MS detection using Agilent 6410 QQQ triple-quadrupole LC mass spectrometer in a positive electrospray ionization mode. The nucleosides were quantified using the nucleoside to base ion mass transitions. Quantification was performed by comparison with the standard curve obtained from pure nucleoside standards running at the same batch of samples. The ratio of $5 \mathrm{mC}$ oxidation was calculated based on the calculated concentrations (Supplementary Fig. S4).

Stability assay of glucosylated 5hmC using model dsDNA. $\beta$-GT ( $40 \mathrm{pmol})$ and 9/11 mer $(400 \mathrm{pmol})$ model dsDNA containing a single $5 \mathrm{hmC}$ were added into a $30-\mu \mathrm{l}$ reaction mixture containing $50 \mathrm{mM}$ HEPES ( $\mathrm{pH} 8$ ), $25 \mathrm{mM} \mathrm{MgCl}_{2}$ and $300 \mu \mathrm{M}$ UDP-glucose for $1 \mathrm{~h}$ at $37^{\circ} \mathrm{C}$. The product with glucosylated $5 \mathrm{hmC}$ was purified using the Qiagen DNA purification kit. Subsequently, 40 pmol $\beta$-GT and 200 pmol product DNA were added into a $30 \mu \mathrm{l}$ reaction mixture containing $50 \mathrm{mM}$ HEPES ( $\mathrm{pH} 8$ ), $25 \mathrm{mM} \mathrm{MgCl}_{2}$ and $1 \mathrm{mM}$ UDP for $1 \mathrm{~h}$ at $37^{\circ} \mathrm{C}$. The reactions were monitored by matrix-assisted laser desorption/ionization-time of flight, with the calculated molecular weight and observed molecular weight indicated (Supplementary Fig. S5). No 5hmC was observed, thus indicating that the reaction is not reversible under the assay conditions we typically employed for TAmC-Seq.

TAmC-Seq library generation. 5mC-enriched-genomic DNA ( $25 \mathrm{ng})$ from three independent $5 \mathrm{mC}$ captures or one non-enriched input genomic DNA was endrepaired, adenylated and ligated to an Illumina Genomic DNA Adapters (Genomic DNA adapter oligo mix), according to standard Illumina protocols for ChIP-Seq library construction, maintaining the proper molar ratios of the adapter to insert. Adapter-ligated fragments $\sim 200-350$ bp were gel-purified by $2 \%$ agarose gel electrophoresis and PCR-amplified for 18 PCR cycles. Libraries were checked for quality and quantified using an Agilent 2100 Bioanalyzer DNA 1000 Chip.

TAmC-Seq library sequencing and read processing. TAmC-Seq libraries were sequenced using the Illumina HiScan platform. Cluster generation was performed with Illumina TruSeq cluster kit v2-cBot-HS. Single reads 51-bp sequencing was completed with Illumina TruSeq SBS kit v3-HS. A dedicated PhiX control lane, as well as $1 \%$ PhiX spike in all other lanes, was used for automated matrix and phasing calculations. Image analysis and base calling were performed with the standard Illumina pipeline. FASTQ sequences were aligned to NCBIv1/mm9 with Bowtie v0.17.2 retaining non-duplicate, unique matches to the genome with no more than three mismatches in the first 30 bases. For binned analyses, unique nonduplicate reads from replicate $5 \mathrm{mC}$ captures were concatenated, extended to a length of $200 \mathrm{bp}$ in the direction of the read, counted in 1 or $10 \mathrm{~kb}$ bins, and normalized to the total number of reads in millions. The total number of unique, non-duplicate reads obtained from each replicate experiment and the total number of unique, non-duplicate reads from each approach are listed in Supplementary Table S4. In cases where the number of reads from TAmC-Seq and MeDIP-Seq were normalized before analysis, reads were randomly sampled from the larger TAmC-Seq set to match the number of reads in the MeDIP-Seq set.

MeDIP-Seq data and read processing. Previously published MeDIP-Seq reads ${ }^{30}$ were downloaded from the ENA Short Read Archive (accessions ERR031627 and ERR031630) and the DDBJ Read Archive (accession DRA000030). As reads from both MeDIP-Seq and TAmC-Seq are tags representing larger DNA fragments, and the MeDIP-Seq reads were derived from paired-end sequencing experiments (although TAmC-Seq reads were derived from a single-read experiments), only read one from the MeDIP-Seq data were used for analyses. Thirty-six base pair MeDIP-Seq reads were aligned to NCBIv1/mm9, extended to a length of $200 \mathrm{bp}$ and post processed in an identical manner, parallel with TAmC-Seq sequences as described above.

TAmC-Seq versus MeDIP-Seq concordance analysis. Binned data $(1 \mathrm{~kb})$ were thresholded by the number of read counts in MeDIP-Seq and TAmC-Seq (randomly sampled to match MeDIP-Seq read counts). Bins at or above defined thresholds were extracted for each capture approach and the number of bins in common was determined. For each $5 \mathrm{mC}$ capture approach, the percentage of total $1 \mathrm{~kb}$ bins genome-wide was also tracked at each threshold. Comparison with conventional whole-genome bisulphite sequence data was done using $\mathrm{CpG}$ methylation signals only $(5 \mathrm{mC}+5 \mathrm{hmC})$ derived as previously published as part of ref. 13 with sequence data from ref. 27 (NCBI GEO accession GSE30206). The HMEC and HCC1954 bisulphite sequencing data were also obtained from GEO (accession GSE29069).
CpG-coverage analysis. Genomic positions for each CpG dinucleotide were derived for the genome (NCBIv1/mm9), and the number of post-processed MeDIP-Seq or TAmC-Seq reads covering each $\mathrm{CpG}$ were counted. Both the number of $\mathrm{CpGs}$ covered and the depth of coverage were tracked. For determining $K_{\mathrm{CpG}-\mathrm{Seg}}$, fractions of MeDIP-Seq and TAmC-Seq reads were randomly and progressively sampled at $10 \%$ intervals up to $100 \%$ of reads. The percentage/number of reads required to cover $50 \%$ of all $\mathrm{CpG}$ dinucleotides, assuming greater than this number are methylated, is defined as $K_{\mathrm{CpG}-\mathrm{Seq}}$.

CpG-content analysis. For determining \% $\mathrm{CpG}$ content in concordant and discordant $1 \mathrm{~kb}$ bins, a read threshold of 5 was used, using an equivalent number of MeDIP-Seq and randomly sampled TAmC-Seq reads. Bins specific to TAmC-Seq at or above this threshold were identified as discordant, whereas concordant bins were those in common between MeDIP-Seq and TAmC-Seq. The mean \%CpG content in a set of $1 \mathrm{~kb}$ bins was determined and compared with the genome-wide average using a $Z$-test. For determining $5 \mathrm{mC}$ signals with varying $\% \mathrm{CpG}$ content, $1-\mathrm{kb}$ bin read counts (reads per million) were determined genome-wide, and bins were subsequently stratified by $\% \mathrm{CpG}$ content at $1 \%$ intervals, from 1 to $18 \%$. The mean reads per million for all $1 \mathrm{~kb}$ bins with a given $\% \mathrm{CpG}$ content was then determined for MeDIP-Seq and TAmC-Seq.

Comparative analyses of methods that map DNA methylation. Random forest classification was performed using Matlab (function TreeBagger, OOBPred $=$ on).

\section{References}

1. Law, J. A. \& Jacobsen, S. E. Establishing, maintaining and modifying DNA methylation patterns in plants and animals. Nat. Rev. Genet. 11, 204-220 (2010).

2. Bhutani, N., Burns, D. M. \& Blau, H. M. DNA demethylation dynamics. Cell 146, 866-872 (2011).

3. Wu, S. C. \& Zhang, Y. Active DNA demethylation: many roads lead to Rome Nat. Rev. Mol. Biol. 11, 607-620 (2010).

4. Deaton, A. M. \& Bird, A. CpG islands and the regulation of transcription. Genes Dev. 25, 1010-1022 (2011).

5. Robertson, K. D. \& Wolffe, A. P. DNA methylation in health and disease. Nat Rev. Genet. 1, 11-19 (2000).

6. Esteller, M. Aberrant DNA methylation as a cancer-inducing mechanism. Annu. Rev. Pharmacol. Toxicol. 45, 629-656 (2005).

7. Jones, P. A. \& Baylin, S. B. The fundamental role of epigenetic events in cancer. Nat. Rev. Genet. 3, 415-428 (2002).

8. Beck, S. \& Rakyan, V. K. The methylome: approaches for global DNA methylation profiling. Trends Genet. 24, 231-237 (2008).

9. Jin, S. G., Kadam, S. \& Pfeifer, G. P. Examination of the specificity of DNA methylation profiling techniques towards 5-methylcytosine and 5hydroxymethylcytosine. Nucleic Acids Res. 38, e125 (2010).

10. Huang, Y. et al. The behaviour of 5-hydroxymethylcytosine in bisulfite sequencing. PLoS ONE 5, e8888 (2010).

11. He, Y. F. et al. Tet-mediated formation of 5-carboxylcytosine and its excision by TDG in mammalian DNA. Science 333, 1303-1307 (2011).

12. Ito, S. et al. Tet proteins can convert 5-methylcytosine to 5-formylcytosine and 5-carboxylcytosine. Science 333, 1300-1303 (2011).

13. $\mathrm{Yu}, \mathrm{M}$. et al. Base-resolution analysis of 5-hydroxymethylcytosine in the mammalian genome. Cell 149, 1368-1380 (2012).

14. Booth, M. J. et al. Quantitative sequencing of 5-methylcytosine and 5hydroxymethylcytosine at single-base resolution. Science 336, 934-937 (2012)

15. Lister, R. et al. Highly integrated single-base resolution maps of the epigenome in Arabidopsis. Cell 133, 523-536 (2008).

16. Lister, R. et al. Human DNA methylomes at base resolution show widespread epigenomic differences. Nature 462, 315-322 (2009).

17. Cokus, S. J. et al. Shotgun bisulphite sequencing of the Arabidopsis genome reveals DNA methylation patterning. Nature 452, 215-219 (2008).

18. Bock, C. et al. Quantitative comparison of genome-wide DNA methylation mapping technologies. Nat. Biotechnol. 28, 1106-1114 (2010).

19. Jacinto, F. V., Ballestar, E. \& Esteller, M. Methyl-DNA immunoprecipitation (MeDIP): hunting down the DNA methylome. Biotechniques 44, 35-39 (2008)

20. Harris, R. A. et al. Comparison of sequencing-based methods to profile DNA methylation and identification of monoallelic epigenetic modifications. Nat. Biotechnol. 28, 1097-1105 (2010).

21. Irizarry, R. A. et al. The human colon cancer methylome shows similar hypoand hypermethylation at conserved tissue-specific CpG island shores. Nat. Genet. 41, 178-186 (2009).

22. Berman, B. P. et al. Regions of focal DNA hypermethylation and long-range hypomethylation in colorectal cancer coincide with nuclear lamina-associated domains. Nat. Genet. 44, 40-46 (2012).

23. Tahiliani, M. et al. Conversion of 5-methylcytosine to 5-hydroxymethylcytosine in mammalian DNA by MLL partner TET1. Science 324, 930-935 (2009) 
24. Ito, S. et al. Role of Tet proteins in $5 \mathrm{mC}$ to $5 \mathrm{hmC}$ conversion, ES-cell self-renewal and inner cell mass specification. Nature 466, 1129-1133 (2010).

25. Pfaffeneder, T. et al. The discovery of 5-formylcytosine in embryonic stem cell DNA. Angew. Chem. Int. Ed. 50, 7008-7012, 2011).

26. Song, C. X. et al. Selective chemical labeling reveals the genome-wide distribution of 5-hydroxymethylcytosine. Nat. Biotechnol. 29, 68-72 (2011).

27. Pastor, W. A. et al. Genome-wide mapping of 5-hydroxymethylcytosine in embryonic stem cells. Nature 473, 394-397 (2011).

28. Kornberg, S. R., Zimmerman, S. B. \& Kornberg, A. Glucosylation of deoxyribonucleic acid by enzymes from bacteriophage-infected Escherichia coli. J. Biol. Chem. 236, 1487-1493 (1961).

29. Song, C. X. et al. Sensitive and specific single-molecule sequencing of 5hydroxymethylcytosine. Nat. Methods 9, 75-77 (2012).

30. Ficz, G. et al. Dynamic regulation of 5-hydroxymethylcytosine in mouse ES cells and during differentiation. Nature 473, 398-402 (2011).

31. Stadler, M. B. et al. DNA-binding factors shape the mouse methylome at distal regulatory regions. Nature 480, 490-495 (2011).

32. Nair, S. S. et al. Comparison of methyl-DNA immunoprecipitation (MeDIP) and methyl-CpG binding domain (MBD) protein capture for genome-wide DNA methylation analysis reveal CpG sequence coverage bias. Epigenetics 6, 34-44 (2011).

33. Hon, G. C. et al. Global DNA hypomethylation coupled to repressive chromatin domain formation and gene silencing in breast cancer. Genome Res. 22, 246-258 (2012)

34. Ruike, Y., Imanaka, Y., Sato, F., Shimizu, K. \& Tsujimoto, G. Genome-wide analysis of aberrant methylation in human breast cancer cells using methylDNA immunoprecipitation combined with high-throughput sequencing. BMC Genomics 11, 137 (2010).

\section{Acknowledgements}

This study was supported by National Institutes of Health (GM071440 to C.H., NS051630 and MH076090 to P.J., U01 ES017166 to B.R.), a Catalyst Award (C.H. and J.-H.M.) from the Chicago Biomedical Consortium, with support from the Searle Funds at The Chicago Community Trust, the Ludwig Institute for Cancer Research (B.R.) and the Emory Genetics Discovery Fund (P.J.). We thank S.F. Reichard, MA, for editing the manuscript.

\section{Author contributions}

C.H., L.Z., C.-X.S., X.W., B.R. and P.J. designed the experiments; L.Z., M.Y., B.P. and J.-H.M. preformed mouse Tetl insect cell expression and purification; L.Z., M.Y. and X.Y.L. performed the $5 \mathrm{mC}$ oxidation, labelling and pulldown of synthetic templates, as well as genomic DNA samples; K.E.S., G.C.H., H.T. and R.C.S. performed deep sequencing and computational analyses; Q.D. carried out the chemical synthesis; C.H., L.Z., K.E.S., G.C.H., C.-X.S., B.R. and P.J. wrote the paper. All authors discussed the results and commented on the manuscript.

\section{Additional information}

Supplementary Information accompanies this paper at http://www.nature.com/ naturecommunications

Competing financial interests: A patent application (PCT/US12/32489) has been filed for the technology disclosed in this publication.

Reprints and permission information is available online at http://npg.nature.com/ reprintsandpermissions/

How to cite this article: Zhang, L. et al. Tet-mediated covalent labelling of 5-methylcytosine for its genome-wide detection and sequencing. Nat. Commun. 4:1517 doi: $10.1038 /$ ncomms2527 (2012). 